\title{
Verleihung des Gustav-Bucky-Preises 2016
}

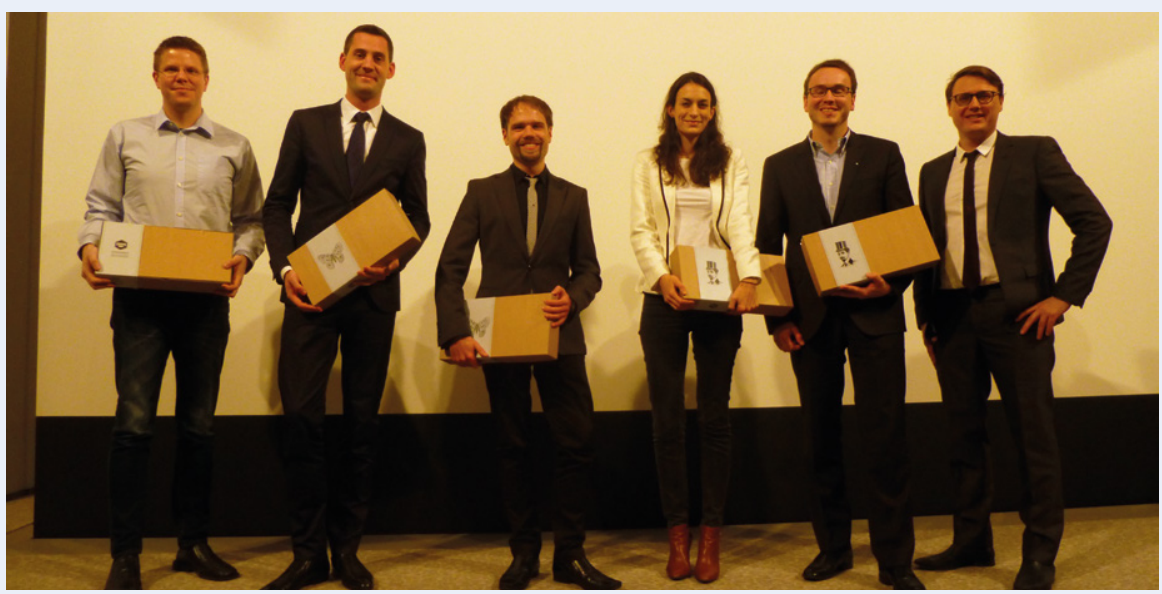

Ein herzliches Dankeschön an die Bewerber für den Gustav-Bucky-Preis 2016.

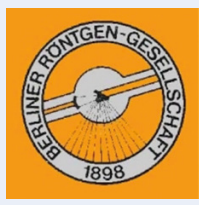

In der letzten wissenschaftlichen Sitzung der Berliner Röntgengesellschaft Röntgenvereinigung zu Berlin und Brandenburg im Jahr 2016 erwartete die Teilnehmenden nochmals ein spannendes Programm, denn auf dem Programm stand: Die Verleihung des Gustav-Bucky-Preises 2016. Sechs nominierte Bewerber aus Berlin und ganz Deutschland hatten ihre Arbeiten eingereicht und freuten sich darauf, diese am 1.11. im Hörsaal der Kaiserin-Friedrich-Stiftung im Herzen Berlins zu präsentieren. Da die Präsentation diesmal auch Teil der Bewertung war, fiel die Entscheidung über die Preisver-

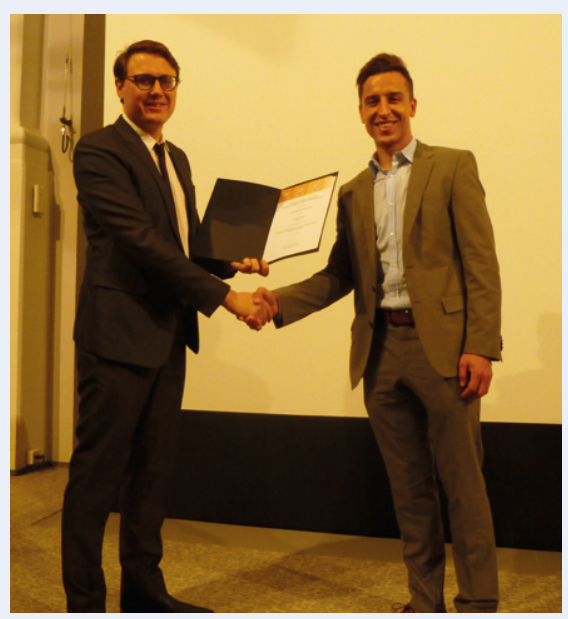

Der Vorsitzende der BRG-RBB, PD Dr. Timm Denecke (li), überreicht die Urkunde des Gustav-Bucky-Preises 2016 an den Gewinner Dr. med. Paul Jahnke. gabe erst an diesem Abend. Die eingereichten höchst innovativen und relevanten Arbeiten und ganz besonders deren Präsentation wurden bei allen Bewerbern hervorragend bewertet und machten die Sitzung zu einem besonderen Abend. Während die Jury, bestehend aus dem Vorstand der BRGRBB, ihre Entscheidung fällte, referierte Frau PD Dr. Diane Renz aus dem Universitätsklinikum Jena einen spannenden Vortrag zum Thema „MRT-Diagnostik der Lunge im Kindesalter“. Danach folgte die feierliche Preisverleihung des Gustav-Bucky-Preises 2016. Wir gratulieren Herrn Dr. Paul Jahnke von der Charité Berlin zum diesjährigen GustavBucky-Preis, den er für seine Arbeit „Radiopaque Three-dimensional Printing: A method to create realistic CT phantoms" erhält. Aber auch die anderen Bewerber gingen nicht leer aus: Jeder erhielt eine erlesen zu- sammengestellte Cocktailbox vom DrinkSyndikat Berlin.

Im Folgenden finden Sie eine Auflistung aller eingereichten Arbeiten zum Gustav-BuckyPreis 2016:

Liver fibrosis and Gd-EOB-DTPA-enhanced MRI: A histopathologic correlation

Dr. Niklas Verloh, Regensburg

Implications of imaging criteria for the management and treatment of intraductual papillary mucinous neoplasms - benign versus malignant findings

Dr. Thula Cannon Walter, Berlin

A novel pairwise Comparison-based method to determine radiation dose reduction potentials of iterative reconstruction algorithms, exemplified through circle of willis computed tomography angiography

Dr. Stephan Ellmann, Erlangen

Exact monitoring of aortic diameters in Marfan patients without gadolinium contrast: intraindividual comparison of 2D SSFP imaging with 3D CE-MRA and echovardiography Dr. Simon Veldhoen, Würzburg

Fractal analysis of the ischemic transition region in chronic ischemic heart disease using magnetic resonance imaging

Florian Michallek, Berlin

Radiopaque Three-dimensional Printing: A method to create realistic CT phantoms Dr. Paul Jahnke, Berlin 